\title{
Asymptotics of the number of eigenvalues of one-term second-order operator equations
}

Ilyas Hashimoglu*

\section{"Correspondence:}

i.hasimoglu@karabuk.edu.tr

Karabuk University, Karabuk, Turkey

\begin{abstract}
We study one-term operator $L$ acting in the space $H_{1}=L_{2}([0, \infty) ; H)$ generated by the operator-differential expression $\mathcal{L}=-\frac{d}{d x}\left(P(x) \frac{d}{d x}\right)$ and the boundary condition $y(0)=0$. We evaluate the asymptotic number of eigenvalues of the operator $L$ under certain conditions.
\end{abstract}

MSC: $34 \mathrm{~K} 08$

Keywords: operator equations; eigenvalues; Hilbert space

\section{Introduction}

\subsection{Related work}

The theory of operator-differential equations with unbounded operator-coefficients is a common tool for the study of infinite systems of ordinary differential equations, partial differential equations and integro-differential equations. The main task in this theory is to determine the behavior of the eigenvalues and eigenfunctions of the associated differential operator. The first significant investigation in this direction belongs to Kostyuchenko and Levitan [1]. They studied the asymptotic behavior of the spectrum of Sturm-Liouville operator with operator coefficient. Later, the subject of investigation has been developed by Gorbachuk [2], Gorbachuk and Gorbachuk [3, 4], Otelbayev [5], Solomyak [6], Maksudov et al. [7], Adiguzelov et al. [8] and Vladimirov [9].

In recent years, Maslov [10] has investigated the number of eigenvalues for a Gibbs ensemble of self-adjoint operators. Muminov [11] has studied the expression for the number of eigenvalues of a Friedrichs model. Also, Vladimirov [12] has calculated the eigenvalues of the Sturm-Liouville problem with a fractal indefinite weight.

\subsection{Formulation of the problem}

Let $L$ denote the differential operator in the space $H_{1}=L_{2}([0, \infty) ; H)$ generated by the operator-differential expression

$$
\mathcal{L}=-\frac{d}{d x}\left(P(x) \frac{d}{d x}\right)
$$

with the boundary condition

$$
y(0)=0
$$

where $P(x)(0 \leq x<\infty)$ is a self-adjoint operator function in a Hilbert space $H$.

(c) 2015 Hashimoglu. This article is distributed under the terms of the Creative Commons Attribution 4.0 International License (http://creativecommons.org/licenses/by/4.0/), which permits unrestricted use, distribution, and reproduction in any medium, provided you give appropriate credit to the original author(s) and the source, provide a link to the Creative Commons license, and indicate if changes were made. 
In this paper, we suppose that the operator $L$ has a discrete spectrum. For instance, in [13], the authors present some conditions under which the operator $L$ has a discrete spectrum.

The aim of the present paper is to study the asymptotic behavior of the eigenvalues of the operator $L$. The existing methods still are not capable to evaluate the number of eigenvalues of the operator $L$ directly. The reason is as follows. It is impossible to apply Courant's variational principle [14] directly because on a finite interval the operator, generated by the differential expression $\mathcal{L}$ and Neumann boundary conditions, has an infinite number of eigenvalues. (For example, 0 is an eigenvalue of infinite multiplicity.) In order to avoid this difficulty, we consider instead of the operator $L$ its some relatively compact perturbation $L_{\alpha}=L+P^{\alpha}$, where $P^{\alpha}$ is the $\alpha$ th power of the operator $P(x)$. In this, we base on the study of Marcus and Matsaev [15], where under certain conditions the authors show that the main terms of the asymptotics of the eigenvalues of the operator $L$ and the unbounded operator with a relatively compact perturbation $L_{\alpha}$ are the same.

\section{Main results}

Throughout the paper, we suppose that the operator-valued function $P(x)$ satisfies the following relative compactness (RC) conditions: There exist self-adjoint operators $A \geq$ $E$ (here $E$ denotes the identity operator) and $B \geq E$ with $D(P(x)) \subset D(A)=D(B)$ and $A^{-1}, B^{-1} \in \sigma_{\infty}$ (here $\sigma_{\infty}$ denotes the set of compact operators in $H$ ); local integral functions $q(x) \geq 1, \varphi(x) \geq 1$ and constants $0<\alpha \leq 1 / 2, \beta>0$ such that for any $f \in D(P(x))$ the following inequalities are satisfied:

(a) $q(x)(A f, f) \leq(P(x) f, f) \leq \varphi(x)(B f, f)$;

(b) $\left(B^{\alpha} f, f\right) \leq\left(A^{1-\beta} f, f\right)$;

(c) $\lim _{N \rightarrow \infty} \int_{N}^{\infty} \frac{1}{q(x)} \int_{N}^{x} \varphi^{\alpha}(s) d s d x=0$.

Below we present a range of lemmas, based on which we prove two main theorems. In Lemma 1, under certain conditions we prove that the operator $P^{\alpha}$ is compact with respect to the operator $L$. In Lemmas 2-6 we evaluate the asymptotics of the eigenvalues of $L_{\alpha}=$ $L+P^{\alpha}$, which is the same as for the operator $L$.

First, we prove the following lemma.

Lemma 1 The operator $P^{\alpha}(x)$ in the space $H_{1}$ is compact relative to operator $L$ under $R C$ conditions.

Proof Let us introduce the spaces $L_{2}^{1}(0, N ; P)$ and $L_{2}\left(0, N ; P^{\alpha}\right)$ as a closure of $H$-valued smooth finite functions near $x=0$ and $x=N$ with metrics

$$
\|y\|_{L_{2}^{1}(0, N ; P)}=\int_{0}^{N}\left(P(x) y^{\prime}, y^{\prime}\right) d x
$$

and

$$
\|y\|_{L_{2}\left(0, N ; P^{\alpha}\right)}=\int_{0}^{N}\left(P^{\alpha}(x) y, y\right) d x
$$

respectively.

We need to check the following two assertions to prove Lemma 1: 
1. For any $\varepsilon>0$, there exists a natural number $N(\varepsilon)$ such that for any $N \geq N(\varepsilon)$ and any $y \in D(L)$ the following inequality holds:

$$
\int_{0}^{N}\left(P^{\alpha}(x) y, y\right) d x \leq \int_{0}^{N}\left(P(x) y^{\prime}, y^{\prime}\right) d x .
$$

2. Embedding operators from the space $L_{2}^{1}(0, N ; P)$ to $L_{2}\left(0, N ; P^{\alpha}\right)$ are completely continuous.

To check assertion 1, we will use Lemma 1 from [13].

Lemma [13] For every finite function $y$, defined on $[0, \infty)$ and taken from the domain $D(\mathcal{L})$, the following two inequalities hold:

$$
\begin{aligned}
& \int_{0}^{\infty}\left(\left|\left(y, y^{\prime}\right)\right| / \int_{x}^{\infty} \frac{d t}{\gamma_{1}(t)}\right) d x \leq 2 \int_{0}^{\infty}\left(P(x) y^{\prime}, y^{\prime}\right) d x, \\
& \int_{0}^{\infty}(y, y) d x \leq C \int_{0}^{\infty}\left(y^{(n-1)}, y^{(n)}\right) x^{2 n-1} d x .
\end{aligned}
$$

Let $\gamma_{1} \leq \gamma_{2} \leq \gamma_{3} \leq \cdots \leq \gamma_{n} \leq \cdots$ be the eigenvalues of the operator $B$. Then, using the above lemma from [13], under RC-conditions we obtain the following chain of inequalities:

$$
\begin{aligned}
\int_{N}^{\infty}\left(P^{\alpha}(x) y, y\right) d x & \leq \int_{N}^{\infty} \varphi^{\alpha}(x)\left(B^{\alpha} y, y\right) d x \\
& =\int_{N}^{\infty} \sum_{k=1}^{\infty} \gamma_{k}^{\alpha} \varphi^{\alpha}(x)\left|y_{k}(x)\right|^{2} d x \\
& =\sum_{k=1}^{\infty} \gamma_{k}^{\alpha} \int_{N}^{\infty} \varphi^{\alpha}(x)\left|y_{k}(x)\right|^{2} d x \\
& =2 \sum_{k=1}^{\infty} \gamma_{k}^{\alpha} \int_{N}^{\infty} \varphi^{\alpha}(x)\left|\int_{x}^{\infty} y_{k}^{\prime}(s) y_{k}(s) d s\right| d x \\
& \leq 2 \sum_{k=1}^{\infty} \gamma_{k}^{\alpha} \int_{N}^{\infty} \varphi^{\alpha}(x) \int_{x}^{\infty} \frac{\left|y_{k}^{\prime}(s) y_{k}(s)\right| \int_{s}^{\infty} \frac{1}{q(t)} d t}{\int_{s}^{\infty} \frac{1}{q(t)} d t} d s d x \\
& \leq 2 \sum_{k=1}^{\infty} \int_{N}^{\infty} \varphi^{\alpha}(x) \int_{x}^{\infty} \frac{1}{q(t)} d t d x \int_{N}^{\infty} q(s) \gamma_{k}^{\alpha}\left|y_{k}^{\prime}(s)\right|^{2} d s \\
& =\int_{N}^{\infty} \frac{1}{q(x)} \int_{N}^{x} \varphi^{\alpha}(s) d s d t \int_{N}^{\infty} q(s)\left(B^{\alpha} y^{\prime}, y^{\prime}\right) d s .
\end{aligned}
$$

Since $A \geq E$ and $1-\beta<1$, by using condition (b) of relative compactness, we have $\left(B^{\alpha} f, f\right) \leq$ $\left(A^{1-\beta} f, f\right) \leq(A f, f)$. Then from the above chain of inequalities we obtain

$$
\begin{aligned}
\int_{N}^{\infty}\left(P^{\alpha}(x) y, y\right) d x & \leq \int_{N}^{\infty} \frac{1}{q(x)} \int_{N}^{x} \varphi^{\alpha}(s) d s d t \int_{N}^{\infty} q(s)\left(A y^{\prime}, y^{\prime}\right) d s \\
& \leq \int_{N}^{\infty} \frac{1}{q(x)} \int_{N}^{x} \varphi^{\alpha}(s) d s d x \int_{N}^{\infty}\left(P(x) y^{\prime}, y^{\prime}\right) d x .
\end{aligned}
$$

From these inequalities and part (b) of the RC-conditions we get assertion 1.

To establish assertion 2, we use Lemma 1 from [16]. 
Lemma [16] If the operator function $Q(x)$ is Bochner integrable on the interval $[0, N]$ and its values are the essence of completely continuous operators in $H$, then the embedding operator from $W_{2}^{1}(0, N)$ to $L_{2}(0, N ; Q)$ is completely continuous.

Since the operator $\varphi^{\alpha}(x) A^{-\beta}$ is completely continuous for all $0 \leq x<\infty$, the above lemma from [16] implies that the embedding operator from $L_{2}^{1}(0, N ; E)$ to $L_{2}\left(0, N ; \varphi^{\alpha}(x) A^{-\beta}\right)$ is completely continuous.

If function $u$ is replaced by $u=A^{\frac{1}{2}} y$, we establish the continuity of the embedding operator from $L_{2}^{1}(0, N ; A)$ to $L_{2}\left(0, N ; \varphi^{\alpha}(x) A^{1-\beta}\right)$.

From parts (a) and (b) of the RC-conditions we have

$$
\begin{aligned}
& \int_{0}^{N}\left(A y^{\prime}, y^{\prime}\right) d x \leq \int_{0}^{N}\left(P(x) y^{\prime}, y^{\prime}\right) d x, \\
& \int_{0}^{N}\left(P^{\alpha}(x) y, y\right) d x \leq \int_{0}^{N} \varphi^{\alpha}(x)\left(B^{\alpha} y, y\right) d x \leq \int_{0}^{N} \varphi^{\alpha}(x)\left(A^{1-\beta} y, y\right) d x .
\end{aligned}
$$

To finish the proof of Lemma 1, we use Theorem 17 from [17].

Theorem [17] If a symmetric operator $K$ and a positive operator $K_{1}$ are defined on $D_{A}$ and the inequality $|(K f, f)| \leq\left(K_{1} f, f\right)$ holds for all $f \in D_{A}$, then the complete continuity of the operator $K_{1}$ with respect to $A$ implies the complete continuity of the operator $K$ with respect to $A$. Here $D_{A}$ denotes the domain of definition of the operator $A$.

From the theorem and the last integral inequalities it follows that the embedding operator from $L_{2}^{1}(0, N ; P)$ to $L_{2}\left(0, N ; P^{\alpha}\right)$ is completely continuous, which proves Lemma 1 .

We now turn to the calculation of the asymptotics of the eigenvalues of operator $L_{\alpha}$ generated by the operator-differential expression

$$
\mathcal{L}_{\alpha}(y)=-\left(P(x) y^{\prime}\right)^{\prime}+P^{\alpha}(x) y
$$

and the boundary condition

$$
y(0)=0 .
$$

Let $\gamma_{1}(x) \leq \gamma_{2}(x) \leq \cdots \leq \gamma_{n}(x) \leq \cdots$ be the family of eigenvalues of the operator function $P(x)$. Suppose that the following conditions are satisfied:

(i) $\gamma_{1}(x) \geq C_{1} x^{5+\delta}$ for large $x$, where $C_{1}>0$ and $\delta>0$.

(ii) $P\left(x_{1}\right) \leq P\left(x_{2}\right)$ for $x_{1}<x_{2}$.

(iii) There exists a positive number $m>0$ such that $\frac{1}{2+\delta}+m<\frac{1}{2}$ and $P^{-\alpha}(0) \in \sigma_{m}$, where $\sigma_{m}=\left\{K \in \sigma_{\infty} \mid \operatorname{tr}\left(\left(K^{*} K\right)^{m / 2}\right)<\infty\right\}$ and $K^{*}$ denotes the adjoint operator of $K$.

For our purpose we also need to consider the following operators:

1. Operator $L_{\alpha}^{1}$, acting in the space $L_{2}\left(\left[\lambda \frac{1}{2+\delta}, \infty\right) ; H\right)$, generated by expression (3) and the boundary condition

$$
y^{\prime}\left(\lambda^{\frac{1}{2+\delta}}\right)=0
$$


2. Operators $L_{\alpha}^{\mathrm{I}}$ and $L_{\alpha}^{\mathrm{II}}$, acting in the space $L_{2}\left(\left[\lambda^{\frac{1}{2+\delta}}, \infty\right) ; H\right)$, generated by expression (3) and the boundary conditions

$$
\begin{aligned}
& y(0)=y\left(\lambda^{\frac{1}{2+\delta}}\right)=0, \\
& y^{\prime}(0)=y^{\prime}\left(\lambda^{\frac{1}{2+\delta}}\right)=0,
\end{aligned}
$$

respectively.

3. Operators $L_{\alpha_{i}}^{\mathrm{I}}$ and $L_{\alpha_{i}}^{\mathrm{II}}$ acting in the space $L_{2}\left(\left[x_{i-1}, x_{i}\right] ; H\right)$ and generated by expression (3) and the boundary conditions

$$
\begin{aligned}
& y\left(x_{i-1}\right)=y\left(x_{i}\right)=0, \\
& y^{\prime}\left(x_{i-1}\right)=y^{\prime}\left(x_{i}\right)=0,
\end{aligned}
$$

respectively.

Lemma 2 If the function $\gamma_{1}(x)$ satisfies (i), the intersection of the set (interval) $(0, \lambda)$ with the spectrum of the operator $L_{\alpha}^{1}$ is empty.

Proof After some algebra (one can find the details in [13], in the proof of Lemma 2), it can be shown that

$$
\int_{0}^{N}(y, y) d x \leq N \int_{0}^{N} \frac{1}{\gamma_{1}(x)} d x \int_{0}^{N}\left(P(x) y^{\prime}, y^{\prime}\right) d x
$$

From this inequality and condition (i) we obtain

$$
\int_{\lambda \frac{1}{2+\delta}}^{\infty}\left(P(x) y^{\prime}, y^{\prime}\right) d x \geq C_{1}(4+\delta) \lambda^{1+\frac{1}{2+\delta}} \int_{\lambda \frac{1}{2+\delta}}^{\infty}(y, y) d x>\lambda \int_{\lambda \frac{1}{2+\delta}}^{\infty}(y, y) d x
$$

The last inequality holds for large $\lambda$. Lemma 2 is proved.

Let $\lambda$ be some positive number. Denote by $N_{\alpha}(\lambda), N_{\alpha}^{\mathrm{I}}(\lambda)$ and $N_{\alpha}^{\mathrm{II}}(\lambda)$ the numbers of eigenvalues of operators $L_{\alpha}, L_{\alpha}^{\mathrm{I}}$ and $L_{\alpha}^{\mathrm{II}}$, respectively, which are less than or equal to $\lambda$. Taking into account Courant's variation principles [14], we find that

$$
N_{\alpha}^{\mathrm{I}}(\lambda) \leq N_{\alpha}(\lambda) \leq N_{\alpha}^{\mathrm{II}}(\lambda)
$$

Let us split the interval $\left[0, \lambda^{\frac{1}{2+\delta}}\right]$ into subintervals of equal length $\omega$. Let $M$ be the number of the created subintervals, and $0=x_{0}<x_{1}<\cdots<x_{M}=\lambda^{\frac{1}{2+\delta}}$.

Lemma 3 If the operator function $P(x)$ satisfies condition (ii) for any $x_{1}<x_{2}$, then for large $\lambda$ the following inequality is valid:

$$
n_{\alpha_{i}}^{\mathrm{II}} \leq \sum_{\gamma_{j}^{\alpha}\left(x_{i-1}\right) \leq \lambda}\left\{\frac{1}{\pi} \int_{x_{i-2}}^{x_{i-1}} \sqrt{\frac{\lambda-\gamma_{j}^{\alpha}(x)}{\gamma_{j}(x)}} d x+1\right\}
$$

where $n_{\alpha_{i}}^{\mathrm{II}}$ is the number of eigenvalues of $L_{\alpha_{i}}^{\mathrm{II}}$, which are less than or equal to $\lambda$, and $i=$ $1,2, \ldots, M$. 
Proof Since the operator function $P(x)$ satisfies condition (ii), we have $P\left(x_{i-1}\right)<P(x)$ for all $x \in\left(x_{i-1}, x_{i}\right)$. Therefore, operator $L_{\alpha_{i}}^{\mathrm{II}}$ is not less than $L_{\alpha_{i}}^{* *}$, acting in the space $L_{2}\left(\left[x_{i-1}, x_{i}\right] ; H\right)$, generated by the expression

$$
-\left(P\left(x_{i-1}\right) y^{\prime}\right)^{\prime}+P^{\alpha}\left(x_{i-1}\right) y
$$

and the boundary condition (9).

Let $n_{\alpha_{i}}^{* *}$ be the number of eigenvalues of the operator $L_{\alpha_{i}}^{* *}$, which are less than or equal to $\lambda$. Then

$$
n_{\alpha_{i}}^{\mathrm{II}}<n_{\alpha_{i}}^{* *} .
$$

Eigenvalues of the operator $L_{\alpha_{i}}^{* *}$ are of the form

$$
\gamma_{j}\left(x_{i-1}\right)\left(\frac{\pi k}{x_{i}-x_{i-1}}\right)^{2}+\gamma_{j}^{\alpha}\left(x_{i-1}\right) \quad(k=0,1,2, \ldots, j=1,2, \ldots) .
$$

From here it directly implies that

$$
n_{\alpha_{i}}^{* *} \leq \sum_{\substack{j \\ \gamma_{j}^{\alpha}\left(x_{i-1}\right) \leq \lambda}}\left\{\frac{\omega}{\pi} \sqrt{\frac{\lambda-\gamma_{j}^{\alpha}\left(x_{i-1}\right)}{\gamma_{j}\left(x_{i-1}\right)}}+1\right\} .
$$

It follows from condition (ii) that all eigenvalues $\gamma_{j}(x)$ are monotonically increasing. Therefore, on the interval $\left(x_{i-2}, x_{i-1}\right)$ we have

$$
\gamma_{j}(x) \leq \gamma_{j}\left(x_{i-1}\right) .
$$

Hence,

$$
\omega \sqrt{\frac{\lambda-\gamma_{j}^{\alpha}\left(x_{i-1}\right)}{\gamma_{j}\left(x_{i-1}\right)}} \leq \int_{x_{i-2}}^{x_{i-1}} \sqrt{\frac{\lambda-\gamma_{j}^{\alpha}(x)}{\gamma_{j}(x)}} d x .
$$

From inequalities (11) and (12) and from the last inequality we obtain

$$
n_{\alpha_{i}}^{\mathrm{II}} \leq \sum_{\substack{j \\ \gamma_{j}^{\alpha}\left(x_{i-1}\right) \leq \lambda}}\left\{\frac{1}{\pi} \int_{x_{i-2}}^{x_{i-1}} \sqrt{\frac{\lambda-\gamma_{j}^{\alpha}(x)}{\gamma_{j}(x)}} d x+1\right\} .
$$

Lemma 3 is proved.

We denote by $\psi_{j}(\lambda)(j=1,2, \ldots)$ the functions defined by the following equation:

$$
\psi_{j}(\lambda)=\min \left\{\sup _{\gamma_{j}^{\alpha}(x) \leq \lambda}(x), \lambda \frac{1}{2+\delta}\right\}
$$

We prove the next lemma. 
Lemma 4 Under conditions of Lemma 3, the following inequality holds:

$$
N_{\alpha}^{\mathrm{II}}(\lambda) \leq n_{\alpha_{1}}^{\mathrm{II}}+\sum_{\substack{j \\ \gamma_{j}^{\alpha}\left(x_{1}\right) \leq \lambda}}\left\{\int_{0}^{\psi_{j}(\lambda)} \frac{1}{\pi} \sqrt{\frac{\lambda-\gamma_{j}^{\alpha}(x)}{\gamma_{j}(x)}} d x+\frac{\psi_{j}(\lambda)}{\omega}\right\} .
$$

Proof According to Courant's variation principle, we have

$$
N_{\alpha}^{\mathrm{II}}(\lambda) \leq \sum_{i=1}^{M} n_{\alpha_{i}}^{\mathrm{II}} .
$$

By Lemma 3, from this inequality we obtain

$$
N_{\alpha}^{\mathrm{II}}(\lambda) \leq n_{\alpha_{1}}^{\mathrm{II}}+\sum_{i \geq 2} \sum_{\substack{j \\ \gamma_{j}^{\alpha}\left(x_{i-1}\right) \leq \lambda}}\left\{\int_{x_{i-2}}^{x_{i-1}} \frac{1}{\pi} \sqrt{\frac{\lambda-\gamma_{j}^{\alpha}(x)}{\gamma_{j}(x)}} d x+1\right\} .
$$

Furthermore, we have

$$
\begin{aligned}
& \sum_{i \geq 2} \sum_{\substack{j \\
\gamma_{j}^{\alpha}\left(x_{i-1}\right) \leq \lambda}} \int_{x_{i-2}}^{x_{i-1}} \sqrt{\frac{\lambda-\gamma_{j}^{\alpha}(x)}{\gamma_{j}(x)}} d x \\
& \quad=\sum_{j} \sum_{i \geq 2} \int_{x_{i-2}}^{x_{i-1}} \sqrt{\frac{\lambda-\gamma_{j}^{\alpha}(x)}{\gamma_{j}(x)}} d x=\sum_{\gamma_{j}^{\alpha}\left(x_{1}\right) \leq \lambda} \int_{0}^{x_{j}^{0}} \sqrt{\frac{\lambda-\gamma_{j}^{\alpha}(x)}{\gamma_{j}(x)}} d x,
\end{aligned}
$$

where

$$
x_{j}^{0}=\min \left\{\max _{\gamma_{j}^{\alpha}\left(x_{i-1}\right) \leq \lambda}\left(x_{i-1}\right), \lambda^{\frac{1}{2+\delta}}\right\} .
$$

On the other hand, $x_{j}^{0} \leq \psi_{j}(\lambda)$, so

$$
\sum_{i \geq 2} \sum_{\substack{j \\ \gamma_{j}^{\alpha}\left(x_{i-1}\right) \leq \lambda}} \int_{x_{i-2}}^{x_{i-1}} \sqrt{\frac{\lambda-\gamma_{j}^{\alpha}(x)}{\gamma_{j}(x)}} d x<\sum_{\substack{j \\ \gamma_{j}^{\alpha}\left(x_{1}\right) \leq \lambda}} \int_{0}^{\psi_{j}(\lambda)} \sqrt{\frac{\lambda-\gamma_{j}^{\alpha}(x)}{\gamma_{j}(x)}} d x .
$$

Taking into account (15), we estimate the sum

$$
\begin{aligned}
\sum_{i \geq 2} \sum_{\substack{j \\
\gamma_{j}^{\alpha}\left(x_{i-1}\right) \leq \lambda}} 1 & =\sum_{j} \sum_{\substack{i \geq 2 \\
\gamma_{j}^{\alpha}\left(x_{i-1}\right) \leq \lambda}} 1 \\
& =\frac{1}{\omega} \sum_{j} \sum_{\substack{i \geq 2 \\
\gamma_{j}^{\alpha}\left(x_{i-1}\right) \leq \lambda}}\left(x_{i-1}-x_{i-2}\right) \\
& =\frac{1}{\omega} \sum_{\substack{j \\
\gamma_{j}^{\alpha}\left(x_{1}\right) \leq \lambda}} x_{j}^{0} \leq \frac{1}{\omega} \sum_{\substack{j \\
\gamma_{j}^{\alpha}\left(x_{1}\right) \leq \lambda}} \psi_{j}(\lambda) .
\end{aligned}
$$


From inequalities (14), (16) and the last inequality we obtain

$$
N_{\alpha}^{\mathrm{II}}(\lambda) \leq n_{1}^{\mathrm{II}}+\sum_{\substack{j \\ \gamma_{j}^{\alpha}\left(x_{1}\right) \leq \lambda}}\left\{\int_{0}^{\psi_{j}(\lambda)} \frac{1}{\pi} \sqrt{\frac{\lambda-\gamma_{j}^{\alpha}(x)}{\gamma_{j}(x)}} d x+\frac{\psi_{j}(\lambda)}{\omega}\right\} .
$$

Lemma 4 is proved.

Lemma 5 Under the conditions of Lemma 3 the following inequality holds:

$$
n_{\alpha_{i}}^{\mathrm{I}} \geq \sum_{\gamma_{j}^{\alpha}\left(x_{i}\right) \leq \lambda}\left\{\frac{1}{\pi} \int_{x_{i}}^{\varphi_{i, j}(\lambda)} \sqrt{\frac{\lambda-\gamma_{j}^{\alpha}(x)}{\gamma_{j}(x)}} d x-1\right\}
$$

where $\varphi_{i, j}(\lambda)=\min \left\{x_{i+1}, \psi_{j}(\lambda)\right\}$ and $i=1,2, \ldots, M$.

Proof Since by our assumption the operator function $P(x)$ increases, then $P(x)<P\left(x_{i}\right)$ on the interval $\left(x_{i-1}, x_{i}\right)$, which implies that the operator $L_{\alpha_{i}}^{\mathrm{I}}$ is not greater than the operator $L_{\alpha_{i}}^{*}$ acting in the space $L_{2}\left(\left[x_{i-1}, x_{i}\right] ; H\right)$ and generated by the expression

$$
-\left(P\left(x_{i}\right) y^{\prime}\right)^{\prime}+P^{\alpha}\left(x_{i}\right) y
$$

and boundary condition (8). In this case

$$
n_{\alpha_{i}}^{\mathrm{I}}>n_{\alpha_{i}}^{*}
$$

where $n_{\alpha_{i}}^{*}$ is the number of eigenvalues of the operator $L_{\alpha_{i}}^{*}$, which are less than or equal to $\lambda$. Eigenvalues of the operator $L_{\alpha_{i}}^{*}$ are of the form

$$
\gamma_{j}\left(x_{i}\right)\left(\frac{\pi k}{x_{i}-x_{i-1}}\right)^{2}+\gamma_{j}^{\alpha}\left(x_{i}\right), \quad \text { where } k=1,2, \ldots \text { and } j=1,2, \ldots
$$

From the inequality

$$
\gamma_{j}\left(x_{i}\right)\left(\frac{\pi k}{x_{i}-x_{i-1}}\right)^{2}+\gamma_{j}^{\alpha}\left(x_{i}\right) \leq \lambda,
$$

it follows that

$$
n_{\alpha_{i}}^{*}=\sum_{\gamma_{j}^{\alpha}\left(x_{i}\right) \leq \lambda}\left\{\frac{\omega}{\pi} \sqrt{\frac{\lambda-\gamma_{j}^{\alpha}(x)}{\gamma_{j}(x)}}\right\} \geq \sum_{\substack{j \\ \gamma_{j}^{\alpha}\left(x_{i}\right) \leq \lambda}}\left\{\frac{\omega}{\pi} \sqrt{\frac{\lambda-\gamma_{j}^{\alpha}\left(x_{i}\right)}{\gamma_{j}\left(x_{i}\right)}}-1\right\},
$$

where $\omega=x_{i}-x_{i-1}$.

Since the function $\gamma_{j}(x)$ monotonically increases, it is clear that when $\gamma_{j}^{\alpha}\left(x_{i+1}\right)<\lambda$, in other words, when $x_{i+1}<\psi_{j}(\lambda)$,

$$
\omega \sqrt{\frac{\lambda-\gamma_{j}^{\alpha}\left(x_{i}\right)}{\gamma_{j}\left(x_{i}\right)}}=\int_{x_{i}}^{x_{i+1}} \sqrt{\frac{\lambda-\gamma_{j}^{\alpha}\left(x_{i}\right)}{\gamma_{j}\left(x_{i}\right)}} d x \geq \int_{x_{i}}^{x_{i+1}} \sqrt{\frac{\lambda-\gamma_{j}^{\alpha}(x)}{\gamma_{j}(x)}} d x
$$


and when $x_{i} \leq \psi_{j}(\lambda) \leq x_{i+1}$,

$$
\omega \sqrt{\frac{\lambda-\gamma_{j}^{\alpha}\left(x_{i}\right)}{\gamma_{j}\left(x_{i}\right)}} \geq \int_{x_{i}}^{\psi_{j}(\lambda)} \sqrt{\frac{\lambda-\gamma_{j}^{\alpha}\left(x_{i}\right)}{\gamma_{j}\left(x_{i}\right)}} d x \geq \int_{x_{i}}^{\psi_{j}(\lambda)} \sqrt{\frac{\lambda-\gamma_{j}^{\alpha}(x)}{\gamma_{j}(x)}} d x .
$$

Thus, we see that, for $\gamma_{j}^{\alpha}\left(x_{i+1}\right) \leq \lambda$,

$$
\omega \sqrt{\frac{\lambda-\gamma_{j}^{\alpha}\left(x_{i}\right)}{\gamma_{j}(x)}} \geq \int_{x_{i}}^{\varphi_{i, j}(\lambda)} \sqrt{\frac{\lambda-\gamma_{j}^{\alpha}\left(x_{i}\right)}{\gamma_{j}(x)}} d x,
$$

where $\varphi_{i, j}(\lambda)=\min \left\{x_{i+1}, \psi_{j}(\lambda)\right\}$. From inequalities (17), (18) and from the last inequality we find

$$
n_{\alpha_{i}}^{\mathrm{I}} \geq \sum_{\gamma_{j}^{\alpha}\left(x_{i}\right) \leq \lambda}\left\{\frac{1}{\pi} \int_{x_{i}}^{\varphi_{i, j}(\lambda)} \sqrt{\frac{\lambda-\gamma_{j}^{\alpha}(x)}{\gamma_{j}(x)}} d x-1\right\} .
$$

Lemma 5 is proved.

Lemma 6 Let the operator function $P(x)$ satisfy conditions (ii) and (iii) for any $x_{1}<x_{2}$. Then the following inequality holds:

$$
N_{\alpha}^{\mathrm{I}}(\lambda) \geq \sum_{j}\left\{\frac{1}{\pi} \int_{\varphi_{0, j}(\lambda)}^{\psi_{j}(\lambda)} \sqrt{\frac{\lambda-\gamma_{j}^{\alpha}(x)}{\gamma_{j}(x)}} d x-\frac{\psi_{j}(\lambda)}{\omega}\right\},
$$

where $\varphi_{0, j}(\lambda)=\min \left\{\omega, \psi_{j}(\lambda)\right\}$.

Proof By Courant's variation principles and Lemma 5, we have

$$
\begin{aligned}
N_{\alpha_{i}}^{\mathrm{I}} & \geq \sum_{i=1}^{M} n_{\alpha_{i}}^{\mathrm{I}} \geq \sum_{i \geq 1} \sum_{\substack{j \\
\gamma_{j}^{\alpha}\left(x_{i}\right) \leq \lambda}}\left\{\frac{1}{\pi} \int_{x_{i}}^{\varphi_{i, j}(\lambda)} \sqrt{\frac{\lambda-\gamma_{j}^{\alpha}(x)}{\gamma_{j}(x)}} d x-1\right\} \\
& =\sum_{j} \sum_{i \geq 1}\left\{\frac{1}{\pi} \int_{x_{i}}^{\varphi_{i, j}(\lambda)} \sqrt{\frac{\lambda-\gamma_{j}^{\alpha}(x)}{\gamma_{j}(x)}} d x-1\right\} .
\end{aligned}
$$

Let us estimate the first term on the right-hand side. Given that $\varphi_{i, j}(\lambda)$ is of the form $\varphi_{i, j}(\lambda)=$ $\min \left\{x_{i+1}, \psi_{j}(\lambda)\right\}$, we get

$$
\begin{aligned}
& \sum_{j} \sum_{i \geq 1} \frac{1}{\pi} \int_{x_{i}}^{\varphi_{i j}(\lambda)} \sqrt{\frac{\lambda-\gamma_{j}^{\alpha}(x)}{\gamma_{j}(x)}} d x \\
& \quad=\frac{1}{\pi} \sum_{j}\left\{\int_{x_{1}}^{x_{2}} \sqrt{\frac{\lambda-\gamma_{j}^{\alpha}(x)}{\gamma_{j}(x)}} d x+\int_{x_{2}}^{x_{3}} \sqrt{\frac{\lambda-\gamma_{j}^{\alpha}(x)}{\gamma_{j}(x)}} d x+\cdots+\int_{x_{i_{0}}}^{\psi_{j}(\lambda)} \sqrt{\frac{\lambda-\gamma_{j}^{\alpha}(x)}{\gamma_{j}(x)}} d x\right\} \\
& \quad=\sum_{\psi_{j}(\lambda) \geq x_{1}} \frac{1}{\pi} \int_{x_{1}}^{\psi_{j}(\lambda)} \sqrt{\frac{\lambda-\gamma_{j}^{\alpha}(x)}{\gamma_{j}(x)}} d x
\end{aligned}
$$


where $x_{i_{0}}$ satisfies the condition $x_{i_{0}} \leq \psi_{j}(\lambda) \leq x_{i_{0}+1}$. Furthermore,

$$
\begin{aligned}
& \sum_{\psi_{j}(\lambda) \geq x_{1}} \frac{1}{\pi} \int_{x_{1}}^{\psi_{j}(\lambda)} \sqrt{\frac{\lambda-\gamma_{j}^{\alpha}(x)}{\gamma_{j}(x)}} d x \\
& =\frac{1}{\pi} \sum_{\psi_{j}(\lambda) \geq x_{1}}\left\{\int_{0}^{\psi_{j}(\lambda)} \sqrt{\frac{\lambda-\gamma_{j}^{\alpha}(x)}{\gamma_{j}(x)}} d x-\int_{0}^{x_{1}} \sqrt{\frac{\lambda-\gamma_{j}^{\alpha}(x)}{\gamma_{j}(x)}} d x\right\} \\
& =\frac{1}{\pi} \sum_{j} \int_{0}^{\psi_{j}(\lambda)} \sqrt{\frac{\lambda-\gamma_{j}^{\alpha}(x)}{\gamma_{j}(x)}} d x-\frac{1}{\pi} \sum_{\psi_{j}(\lambda) \leq x_{1}} \int_{0}^{\psi_{j}(\lambda)} \sqrt{\frac{\lambda-\gamma_{j}^{\alpha}(x)}{\gamma_{j}(x)}} d x \\
& \quad-\frac{1}{\pi} \sum_{\psi_{j}(\lambda) \geq x_{1}} \int_{0}^{x_{1}} \sqrt{\frac{\lambda-\gamma_{j}^{\alpha}(x)}{\gamma_{j}(x)}} d x \\
& =\frac{1}{\pi} \sum_{j}\left\{\int_{0}^{\psi_{j}(\lambda)} \sqrt{\frac{\lambda-\gamma_{j}^{\alpha}(x)}{\gamma_{j}(x)}} d x-\int_{0}^{\varphi_{0, j}(\lambda)} \sqrt{\frac{\lambda-\gamma_{j}^{\alpha}(x)}{\gamma_{j}(x)}} d x\right\} \\
& =\frac{1}{\pi} \sum_{j} \int_{\varphi_{0, j}(\lambda)}^{\psi_{j}(\lambda)} \sqrt{\frac{\lambda-\gamma_{j}^{\alpha}(x)}{\gamma_{j}(x)}} d x .
\end{aligned}
$$

From inequalities (21) and (22) we find

$$
\begin{aligned}
& \sum_{j} \sum_{i \geq 1} \frac{1}{\pi} \int_{x_{i}}^{\varphi_{i, j}(\lambda)} \sqrt{\frac{\lambda-\gamma_{j}^{\alpha}(x)}{\gamma_{j}(x)}} d x \\
& =\frac{1}{\pi} \int_{\varphi_{0, j}(\lambda)}^{\psi_{j}(\lambda)}\left(\sum_{j} \sqrt{\frac{\lambda-\gamma_{j}^{\alpha}(x)}{\gamma_{j}(x)}} d x\right) .
\end{aligned}
$$

For the second term on the right-hand side of (20), as before (in the proof of Lemma 4), we have

$$
\sum_{i \geq 2} \sum_{j} 1 \leq \frac{1}{\omega} \sum_{j} \psi_{j}(\lambda)
$$

Finally, taking into account (21), (24) and (25), we obtain the desired inequality (20). Lemma 6 is proved.

Corollary Under the conditions of Lemma 6, the following inequality holds:

$$
N_{\alpha_{i}}^{\mathrm{I}} \geq \frac{1}{\pi} \sum_{j} \int_{0}^{\psi_{j}(\lambda)} \sqrt{\frac{\lambda-\gamma_{j}^{\alpha}(x)}{\gamma_{j}(x)}} d x-\sqrt{\lambda} \omega c_{1}-\frac{\psi_{1}(\lambda)}{\omega} l_{\lambda},
$$

where $c_{1}$ is a constant and $l_{\lambda}$ is the number of eigenvalues of the operator $P^{\alpha}(0)$, which are less than or equal to $\lambda$, i.e.,

$$
l_{\lambda}=\sum_{\gamma_{j}^{\alpha}(0) \leq \lambda} 1 .
$$


Proof In fact, by Lemma 6

$$
\begin{aligned}
N_{\alpha}^{\mathrm{I}}(\lambda) \geq & \frac{1}{\pi} \sum_{j} \int_{0}^{\psi_{j}(\lambda)} \sqrt{\frac{\lambda-\gamma_{j}^{\alpha}(x)}{\gamma_{j}(x)}} d x \\
& -\frac{1}{\pi} \sum_{j} \int_{0}^{\varphi_{0, j}(\lambda)} \sqrt{\frac{\lambda-\gamma_{j}^{\alpha}(x)}{\gamma_{j}(x)}} d x-\frac{1}{\omega} \sum_{j} \psi_{j}(\lambda) .
\end{aligned}
$$

Let us estimate the second term on the right-hand side of this inequality. Since all functions $\gamma_{j}(x)(j=1,2, \ldots)$ monotonically increase on half axis $[0, \infty)$, we have

$$
\begin{aligned}
& \frac{1}{\pi} \sum_{j} \int_{0}^{\varphi_{0, j}(\lambda)} \sqrt{\frac{\lambda-\gamma_{j}^{\alpha}(x)}{\gamma_{j}(x)}} d x \\
& \quad<\frac{1}{\pi} \sum_{j} \int_{0}^{\varphi_{0, j}(\lambda)} \sqrt{\frac{\lambda}{\gamma_{j}(x)}} d x \leq \frac{\sqrt{\lambda}}{\pi} \sum_{\gamma_{j}^{\alpha}(0) \leq \lambda} \int_{0}^{\omega} \frac{1}{\sqrt{\gamma_{j}(x)}} d x \\
& \quad \leq \frac{\omega \sqrt{\lambda}}{\pi} \sum_{\gamma_{j}^{\alpha}(0) \leq \lambda} \frac{1}{\sqrt{\gamma_{j}(0)}} \leq c_{1} \omega \sqrt{\lambda} .
\end{aligned}
$$

For the third term on the right-hand side of (26), we find

$$
\frac{1}{\omega} \sum_{j} \psi_{j}(\lambda) \leq \frac{\psi_{1}(\lambda)}{\omega} \sum_{\gamma_{j}^{\alpha}(0) \leq \lambda} 1=\frac{\psi_{1}(\lambda)}{\omega} l_{\lambda} .
$$

From these inequalities, we obtain inequality (26), which proves the corollary.

Assume that $P^{-\alpha}(0) \in \sigma_{m}$, where $m$ is some positive number satisfying the condition $\frac{1}{2+\delta}+m<\frac{1}{2}$. Then we have

$$
\begin{aligned}
\text { const } & \geq \sum_{\gamma_{j}^{-\alpha}(0) \geq \lambda^{-1}}\left(\gamma_{j}^{-\alpha}(0)\right)^{m} \geq \sum_{\gamma_{j}^{-\alpha}(0) \geq \lambda^{-1}} \lambda^{-m} \\
& \geq \lambda^{-m} \sum_{\gamma_{j}^{-\alpha}(0) \geq \lambda^{-1}} 1=\lambda^{-m} \sum_{\gamma_{j}^{\alpha}(0) \leq \lambda} 1=\lambda^{-m} l_{\lambda} .
\end{aligned}
$$

Hence

$$
l_{\lambda} \leq \text { const } \cdot \lambda^{m} \text {. }
$$

Now take the step $\omega$ as

$$
\omega=\frac{\lambda^{\frac{1}{2+\delta}}}{\left[\lambda^{\frac{1}{2+\delta}+\theta}\right]}, \quad \text { where } \frac{1}{2+\delta}+\theta+m<\frac{1}{2} .
$$

Using this form of $\omega$, we estimate the numbers $N_{\alpha}^{\mathrm{I}}(\lambda)$ and $N_{\alpha}^{\mathrm{II}}(\lambda)$. 
From Lemma 4 it follows that

$$
N_{\alpha}^{\mathrm{II}}(\lambda) \leq n_{\alpha_{1}}^{\mathrm{II}}+\sum_{\substack{j \\ \gamma_{j}^{\alpha}\left(x_{1}\right) \leq \lambda}}\left\{\int_{0}^{\psi_{j}(\lambda)} \frac{1}{\pi} \sqrt{\frac{\lambda-\gamma_{j}^{\alpha}(x)}{\gamma_{j}(x)}} d x+\frac{\psi_{j}(\lambda)}{\omega}\right\} .
$$

Using inequality (29), we estimate the number $n_{\alpha_{1}}^{\mathrm{II}}$,

$$
\begin{aligned}
n_{\alpha_{1}}^{\mathrm{II}} & \leq \sum_{\substack{j \\
\gamma_{j}^{\alpha}(0) \leq \lambda}}\left(\frac{\omega}{\pi} \sqrt{\left.\frac{\lambda-\gamma_{j}^{\alpha}(0)}{\gamma_{j}(0)}+\frac{\psi_{j}(\lambda)}{\omega}\right)}\right. \\
& \leq \frac{\omega \sqrt{\lambda}}{\pi} \sum_{\substack{j \\
\gamma_{j}^{\alpha}(0) \leq \lambda}} \frac{1}{\sqrt{\gamma_{j}(0)}}+\sum_{\substack{j \\
\gamma_{j}^{\alpha}(0) \leq \lambda}} 1 \leq c_{2} \lambda^{\frac{1}{2}-\theta}+c_{3} \lambda^{m+\theta},
\end{aligned}
$$

where $c_{2} \geq \frac{1}{\pi} \operatorname{tr}\left(P^{-\frac{1}{2}}(0)\right)$ and $c_{3} \geq \operatorname{tr}\left(P^{-\alpha m}(0)\right)$ (here, $\operatorname{tr}(A)$ denotes the trace of the operator $A)$.

It follows from inequalities (31) and (32) and formula (13) that

$$
\begin{aligned}
N_{\alpha}^{\mathrm{II}}(\lambda) & \leq \sum_{j} \frac{1}{\pi} \int_{0}^{\psi_{j}(\lambda)} \sqrt{\frac{\lambda}{\gamma_{j}(x)}} d x+\frac{\psi_{1}(\lambda)}{\omega} l_{\lambda}+c_{2} \lambda^{\frac{1}{2}-\theta}+c_{3} \lambda^{m} \\
& \leq \sqrt{\lambda} \sum_{j} \frac{1}{\pi} \int_{0}^{\psi_{j}(\lambda)} \frac{1}{\sqrt{\gamma_{j}(x)}} d x+c_{1} \lambda^{\frac{1}{2+\delta}+m+\theta}+c_{2} \lambda^{\frac{1}{2}-\theta}+c_{3} \lambda^{m} .
\end{aligned}
$$

Using the corollary to Lemma 6, inequality (29) and formula (30), for $N_{\alpha}^{\mathrm{I}}(\lambda)$, we obtain the following inequality:

$$
N_{\alpha}^{\mathrm{I}}(\lambda) \geq \frac{1}{\pi} \sum_{j} \int_{0}^{\psi_{j}(\lambda)} \sqrt{\frac{\lambda-\gamma_{j}^{\alpha}(x)}{\gamma_{j}(x)}} d x-c_{4} \lambda^{\frac{1}{2}-\theta}-c_{3} \lambda^{\frac{1}{2+\delta}+m+\theta} .
$$

Let us estimate the first term on the right-hand side:

$$
\begin{aligned}
& \frac{1}{\pi} \sum_{j} \int_{0}^{\psi_{j}(\lambda)} \sqrt{\frac{\lambda-\gamma_{j}^{\alpha}(x)}{\gamma_{j}(x)} d x} \\
& \quad=\frac{1}{\pi} \sum_{j} \int_{0}^{\psi_{j}(\lambda)} \sqrt{\frac{(\sqrt{\lambda})^{2}-2 \gamma_{j}^{\alpha}(x) \frac{\sqrt{\lambda}}{\sqrt{\lambda}}+\frac{\gamma_{j}^{2 \alpha}(x)}{\lambda}-\frac{\gamma_{j}^{2 \alpha}(x)}{\lambda}+\gamma_{j}^{\alpha}(x)}{\gamma_{j}(x)}} d x \\
& \geq \frac{1}{\pi} \sum_{j} \int_{0}^{\psi_{j}(\lambda)} \sqrt{\frac{(\sqrt{\lambda})^{2}-2 \gamma_{j}^{\alpha}(x) \frac{\sqrt{\lambda}}{\sqrt{\lambda}}+\frac{\gamma_{j}^{2 \alpha}(x)}{\lambda}}{\gamma_{j}(x)}} d x \\
& =\frac{1}{\pi} \sum_{j} \int_{0}^{\psi_{j}(\lambda)} \frac{\sqrt{\lambda}-\frac{\gamma_{j}^{\alpha}(x)}{\sqrt{\lambda}}}{\sqrt{\gamma_{j}(x)}} d x \geq \frac{1}{\pi} \sum_{j} \sqrt{\lambda} \int_{0}^{\psi_{j}(\lambda)} \frac{1}{\sqrt{\gamma_{j}(x)}} d x-c_{6} \psi_{1}(\lambda) .
\end{aligned}
$$


From inequalities (34) and (35) we obtain

$$
N_{\alpha}^{\mathrm{I}}(\lambda) \geq \frac{\sqrt{\lambda}}{\pi} \sum_{j} \int_{0}^{\psi_{j}(\lambda)} \frac{1}{\sqrt{\gamma_{j}(x)}} d x-c_{1}^{\mathrm{I}} \lambda \frac{1}{2+\delta}-c_{2}^{\mathrm{I}} \lambda^{\frac{1}{2}-\theta}-c_{3}^{\mathrm{I}} \lambda^{\frac{1}{2+\delta}+m+\theta} .
$$

By Lemma 4 and inequalities (33) and (36), we obtain

$$
\begin{aligned}
& \frac{1}{\pi} \sum_{j} \int_{0}^{\psi_{j}(\lambda)} \frac{1}{\sqrt{\gamma_{j}(x)}} d x-c_{0} \lambda^{-\frac{1}{2}+\frac{1}{2+\delta}+m+\theta} \\
& \quad \leq \frac{N_{\alpha}(\lambda)}{\sqrt{\lambda}} \leq \frac{1}{\pi} \sum_{j} \int_{0}^{\psi_{j}(\lambda)} \frac{1}{\sqrt{\gamma_{j}(x)}} d x+c_{1} \lambda^{-\frac{1}{2}+\frac{1}{2+\delta}+m+\theta} .
\end{aligned}
$$

Given that $-\frac{1}{2}+\frac{1}{2+\delta}+m+\theta<0$, we finally obtain the following relation for the number of eigenvalues of the operator $L_{\alpha}$ :

$$
\lim _{\lambda \rightarrow \infty} \frac{N_{\alpha}(\lambda)}{\sqrt{\lambda}}=\frac{1}{\pi} \sum_{j} \int_{0}^{\infty} \frac{1}{\sqrt{\gamma_{j}(x)}} d x .
$$

Thus we have proved the following theorem.

Theorem 1 Let the operator L have discrete spectrum. Then under RC-conditions and (i)(iii), the number $N_{\alpha}(\lambda)$ of eigenvalues of operator $L_{\alpha}$ satisfies relation (37).

The next theorem is Theorem 3.2 in [15] which has been proved by Marcus and Matsaev.

Theorem [15] Let $M$ be a normal operator with discrete spectrum; and all its eigenvalues, which lie in the corner $\psi_{2 \theta}=\{\lambda:|\varphi|<2 \theta\}\left(0<\theta \leq \frac{\pi}{2}\right)$, are positive and their number is infinite. Also let $B$ be an operator, which is compact with respect to $M$, and $A=M+B$. If $\lim _{r \rightarrow \infty ; \varepsilon \rightarrow 0} \frac{N_{+}(r(1+\varepsilon), M)}{N_{+}(r, M)}=1$, then $N(r, \theta, A) \sim N_{+}(r, M)$. Here, $N_{+}(r, M)$ denotes the number of positive eigenvalues of $M$, which are less than or equal to $r$.

Operator $P^{\alpha}$ is compact relative to operator $L_{\alpha}$ and all the conditions of the above theorem are satisfied for operators $M=L_{\alpha}, B=-P^{\alpha}$ and $A=L_{\alpha}-P^{\alpha}$. Then

$$
N_{\lambda}\left(L_{\alpha}\right) \sim N_{\lambda}(L)
$$

By taking into account Lemma 1 and Theorem 3.2 from [15], we obtain the following main theorem.

Theorem 2 Under the conditions of Theorem 1 , the following relation is satisfied for the asymptotics of the eigenvalues of the operator $L$ when $\lambda \rightarrow \infty$ :

$$
N_{\lambda}(L)=\frac{\sqrt{\lambda}}{\pi} \sum_{j}\left(\int_{0}^{\psi_{j}(\lambda)} \frac{1}{\sqrt{\gamma_{j}(x)}} d x+o(1)\right) .
$$

\section{Example}

Example Consider the operator $L$ generated by the differential expression

$$
\mathcal{L} u=(-1)^{k+1} \frac{\partial^{k+1}}{\partial x \partial y^{k}}\left(a(x, y) \frac{\partial^{k+1} u}{\partial y^{k} \partial x}\right)
$$


and the boundary and initial conditions

$$
\begin{aligned}
& \frac{\partial^{j} u}{\partial y^{j}}(x, \pm 1)=0, \quad i=0,1, \ldots, k-1 ; \\
& u(0, y)=0,
\end{aligned}
$$

where $a(x, y) \geq 1$ for all $0 \leq x \leq \infty,-1 \leq y \leq 1$.

This operator can be reduced to the operator generated by the operator-differential expression

$$
\mathcal{L} u=-\frac{d}{d x}\left(P(x) \frac{d}{d x} u\right)
$$

and the boundary condition

$$
u(0)=0,
$$

where

$$
P(x) f=\left\{(-1)^{k+1} \frac{d^{k}}{d y^{k}}\left(a(x, y) \frac{d^{k} f}{d y^{k}}\right) ; \frac{d^{i} f}{d y^{i}}( \pm 1)=0, i=0,1, \ldots, k-1\right\} .
$$

The operator function $P(x)$ acts in the space $L_{2}(-1,1)$ for all $x$.

Consider the following functions:

$$
\begin{aligned}
& a_{1}(x)=\min _{-1 \leq y \leq 1} a(x, y), \\
& a_{2}(x)=\max _{-1 \leq y \leq 1} a(x, y) .
\end{aligned}
$$

Assume that there exists such a number $0<\alpha \leq \frac{1}{2}$ for which the following condition is satisfied:

(1) $\lim _{N \rightarrow \infty} \int_{N}^{\infty} \frac{1}{a_{1}(x)} \int_{N}^{x} a_{2}^{\alpha}(s) d s d x=0$.

Then the RC-conditions are satisfied, where operators $A$ and $B$ and functions $q(x)$ and $\varphi(x)$ are defined as follows:

$$
\begin{aligned}
& A f=B f=\left\{(-1)^{k} \frac{d^{2 k}}{d y^{2 k}} f ; \frac{d^{i} f}{d y^{i}}( \pm 1)=0, i=0,1, \ldots, k-1\right\}, \\
& q(x)=a_{1}(x), \quad \varphi(x)=a_{2}(x) .
\end{aligned}
$$

Let the function $a(x, y)$ and the order $k$ of differential operator $P(x)$ satisfy the following conditions:

(2) $a_{1}(x) \geq c x^{5+\delta}$.

(3) The function $a(x, y)$ is monotonically increasing with respect to the variable $x$.

(4) The order $k$ of differential operator $P(x)$ is such that the condition $\sum_{n=1}^{\infty} \frac{1}{n^{2 m \alpha k}}<\infty$ is satisfied for some number $m$, where $\frac{1}{2+\delta}+m<\frac{1}{2}$. 
Then, for the asymptotics of the number of eigenvalues of the operator $L$, we have the following formula:

$$
N_{\lambda}(L)=\frac{\sqrt{\lambda}}{\pi} \sum_{n=1}^{\infty}\left(\int_{0}^{\infty} \frac{1}{\sqrt{\alpha_{n}(x)}} d x+o(1)\right)
$$

where $\alpha_{n}(x)$ are eigenvalues of the operator $P(x)$.

\section{Competing interests}

The author declares that he has no competing interests.

\section{Author's contributions}

The author performed all tasks of this research: drafting, developing the research topic, writing, and revising the paper. The author has read and approved the final manuscript.

\section{Acknowledgements}

I am grateful to the anonymous reviewers for their comments and suggestions, which have helped to improve the quality of this paper.

\section{Received: 24 June 2015 Accepted: 21 October 2015 Published online: 26 October 2015}

\section{References}

1. Kostyuchenko, AG, Levitan, BM: Asymptotic behavior of the eigenvalues of the Sturm-Liouville operator problem. Funct. Anal. Appl. 1(1), 75-83 (1967)

2. Gorbachuk, ML: Self-adjoint boundary problems for a second-order differential equation with unbounded operator coefficient. Funkc. Anal. Prilozh. 5(1), 10-21 (1971)

3. Gorbachuk, VI, Gorbachuk, ML: On a class of boundary value problems for Sturm-Liouville equation with operator coefficients. Ukr. Math. J. 24(3), 291-305 (1972)

4. Gorbachuk, VI, Gorbachuk, ML: Some problems of spectral theory of differential equations of elliptic type in space of vector functions. Ukr. Math. J. 28(3), 313-324 (1976)

5. Otelbayev, M: On Titcmars method of restriction of resolvent. Dokl. Akad. Nauk SSSR 281(4), 787-790 (1973)

6. Solomyak, MZ: Asymptotics of the spectrum of the Schrodinger operator with non-regular homogeneous potential. Math. USSR Sb. 55(1), 19-37 (1986)

7. Maksudov, FG, Bayramoglu, M, Adiguzelov, EE: On asymptotics of spectrum and trace of high order differential operator with operator coefficients. Doğa Turk. J. Math. 17(2), 113-128 (1993)

8. Adiguzelov, E, Avci, H, Gul, E: An asymptotic formula for the number of eigenvalues of a differential operator. Proyecciones 20(1), 65-82 (2001)

9. Vladimirov, AA: Estimates of the number of eigenvalues of self-adjoint operator functions. Mat. Zametki 74(6), 838-847 (2003)

10. Maslov, VP: On the number of eigenvalues for a Gibbs ensemble of self-adjoint operators. Mat. Zametki 83(3), 465-467 (2008)

11. Muminov, Ml: Expression for the number of eigenvalues of a Friedrichs model. Math. Notes 82(1), 67-74 (2007)

12. Vladimirov, AA: Calculating the eigenvalues of the Sturm-Liouville problem with a fractal indefinite weight. Zh. Vychisl. Mat. Mat. Fiz. 47(8), 1350-1355 (2007)

13. Bayramoglu, M, Hashimov, IF: Discreteness of the spectrum of one-term operator-differential equation of even order. News Acad. Sci. Azerb. SSR, Ser. Phys.-Tech. Math. Sci. 8(1), 19-25 (1987)

14. Courant, R, Hilbert, D: Methods of Mathematical Physics, vol. 1. Wiley-VCH, Weinheim (1989)

15. Marcus, AS, Matsaev, Vl: Comparison theorems for spectra of linear operators and spectral asymptotics. Trans. Mosc. Math. Soc. 45, 133-181 (1982)

16. Yafaev, DR: On negative spectrum of Schrödinger operator equation. Math. Notes 7(6), 753-763 (1970)

17. Glazman, IM: Direct Methods of Qualitative Spectral Analysis of Singular Differential Operators. Fizmatgiz, Moscow (1963) 